anxiety and 38\% with depression, comparable to the average recovery rate $(41.6 \%)$ of physically healthy patients in London IAPT services during the same period. Overall $80 \%$ of patients showed significant improvement in their experience of their COPD, including their perceived control and adjustment to the condition.

Conclusions An integrated clinical psychology service offers significant advantage to traditional mental health services in improving service access, symptoms reduction, and improvement in self-reported quality of life of COPD patients.

\section{M5 VIRTUAL CLINICS FOR CHRONIC LUNG DISEASE- COMPARISON AND LEARNING FROM PATIENT GROUPS IN PRIMARY CARE \& INTEGRATED RESPIRATORY CARE SERVICES}

J Cornwallis, S Purlackee, A Ahmed, R Hassan, G Ng-Man-Kwong. Pennine Lung Service, Royal Oldham Hospital, Manchester, UK

\subsection{6/thoraxjnl-2017-210983.427}

Introduction and Aims The virtual clinic (VC) was designed to identify and review patients with COPD diagnosis and/or FEV1 $\geq 50 \%$ predicted and/or on high dose inhaled corticosteroids (ICS) to recommend interventions to optimise care. Our study piloted VCs across 2 patient groups in primary care (PC) and under the care of our local integrated respiratory care service (IRCS). The aims were to identify patients who would benefit from national guideline based interventions such as modification of inhaler therapies, pulmonary rehabilitation (PR) and/or additional specialist advice.

Results VCs were undertaken in six PC health centres with GP and/or practice nurse and for IRCS group respectively. In total 161 patients were reviewed (PC group 94/161 (68\%) and IRCS 67/161). Mean age 63 years (range 38-96), male 67/161 (42\%), mean\% predicted FEV1 51\% (range 11-117). Overall 98/161 (60\%) had potential change in inhaler therapy identified, PC 55/94 (59\%) and IRCS 43/67 (64\%) respectively ( $\mathrm{p}=0.47 \mathrm{NS}) .40 / 161 \quad(25 \%)$ had recommendation to stop or wean ICS (PC 28/94 (30\%) and IRCS 12/67 (18\%, $\mathrm{p}=0.08 \mathrm{NS}) .47 / 161$ (29\%) recommended referral for PR (PC $38 / 94(40 \%)$ vs IRCS $9 / 67(13 \%), \mathrm{p}<0.01)$. For the PC group $22 / 94(23 \%)$ were referred to IRCS and 3/94 (4\%) were removed from COPD register. 8/161 (5\%) were identified as potentially requiring HRCT investigation, $7 / 94$ (7\%) in the PC group.

Conclusion Virtual Clinics as part of an Integrated Respiratory Care Service can generate important treatment optimisations including inhaler modification in nearly two thirds (trial of ICS cessation in 25\%) and PR referral in one third of reviews (significantly in the PC group). VCs confer patient benefit, support medicines management, PC training and education (including quality assurance) and effective and efficient use of specialist time.

\section{M6 BREATHLESSNESS RAPID EVALUATION, ASSESSMENT, TREATMENT AND HEALTH EDUCATION (BREATHE); A NOVEL APPROACH TO BREATHLESSNESS IN STOCKPORT}

${ }^{1} \mathrm{~V}$ Gupta, ${ }^{2} \mathrm{~K}$ McEwan, ${ }^{3} \mathrm{P}$ Ansbro, ${ }^{1} \mathrm{RR}$ Viswesvaraiah, ${ }^{1} \mathrm{~K}$ Fern, ${ }^{1} \mathrm{H}$ Oxenforth, ${ }^{1} \mathrm{~N}$ Okolie, 1) Thompson, ${ }^{1} \mathrm{~N}$ Davies, ${ }^{1} \mathrm{~K}$ Lewis-Jones, ${ }^{1} \mathrm{~F}$ Poisson, ${ }^{3} \mathrm{~S}$ Gaduzo. ${ }^{1}$ Stockport NHS Foundation Trust, Stockport, UK; ${ }^{2}$ Stockport CCG, Stockport, UK; ${ }^{3}$ Stockport Together, Stockport, UK

\subsection{6/thoraxjnl-2017-210983.428}

Background As part of a collaborative 100 day rapid testing programme with NHS England and Stockport Together, we aimed to improve the way patients with breathlessness are assessed, diagnosed and treated. Between June 2015 and June $20165.2 \%$ and 9.1\% of cardiology and respiratory patients seen at Stockport NHS Foundation Trust respectively were subsequently referred to the other specialty causing delays in diagnosis and multiple clinic attendances. Breathlessness is a common reason for unscheduled presentation to ED and primary care.

Aims To provide a one stop multidisciplinary clinic to step down $75 \%$ patients presenting with breathlessness in 2 visits or less to primary care or appropriate specialist community teams, with clear diagnoses and patient led management plans. Methods A clinic consisting of a cardiologist, respiratory physician, cardiac and respiratory physiologists and a member of The Prevention Alliance (TPA) was set up initially in the community and then in Stepping Hill Hospital. Primary care referrals on existing outpatient waiting lists were used and a GP referral template was also created. In a single day patients had CXR, ECG, ECHO and spirometry prior to seeing both consultants together. TPA supported the patient to explore and develop their own plan and signposted to appropriate $3 \mathrm{rd} \mathrm{sec}$ tor or self-help organisations.

Results Between June 2016 and May 201762 patients were seen in the clinic (28/34 male/female; mean age 67.7 \pm 1.5$)$. To date, $43.5 \%$ patients have been stepped down and a further $19.4 \%$ are awaiting investigations and pending step down within 2 visits. The rest require further follow up with respiratory $(22.6 \%)$, cardiology $(9.6 \%)$ or both specialties $(4.8 \%)$. Patient feedback was extremely positive; with a median rating of 1 (range 1-3) in overall helpfulness (1 being most helpful; 5 least helpful) and multiple comments in favour of the multispeciality clinic.

Conclusion This multi-disciplinary approach to breathlessness has successfully led to a significant number of patients being stepped down from secondary care quickly and efficiently, within 2 visits and with appropriate management plans and education. This model will hopefully prevent these patients being referred to cardiology/respiratory services in the future, reduce attendances to ED and primary care and ensure patient led management. 